\title{
Multidrug-resistant tuberculosis in children: evidence from global surveillance
}

\author{
Matteo Zignol', Charalambos Sismanidis', Dennis Falzon', Philippe Glaziou', \\ Masoud Dara ${ }^{2}$ and Katherine Floyd ${ }^{1}$
}

Affiliations: ${ }^{1}$ STOP TB Dept, World Health Organization, Geneva, Switzerland. ${ }^{2}$ TB and M/XDR-TB Programme, World Health Organization Regional Office for Europe, Copenhagen, Denmark.

Correspondence: M. Zignol, STOP TB Dept, World Health Organization, 20 Avenue Appia, 1211 Geneva 27. Switzerland. E-mail: zignolmawho.int

ABSTRACT Multidrug-resistant tuberculosis (MDR-TB) can affect persons of any age, but it remains unknown whether children are more or less likely than adults to have MDR-TB.

Representative drug resistance surveillance data reported to the World Health Organization between 1994 and 2011 were analysed to test the association between MDR-TB and age group (children aged $<15$ years versus adults aged $\geqslant 15$ years), using odds ratios derived by logistic regression with robust standard errors.

Of 85 countries with data from nationwide surveys or surveillance systems, 35 reported at least one paediatric MDR-TB case. Aggregated data on age and drug susceptibility testing for 323046 tuberculosis cases notified in these 35 countries were analysed. Odds ratios for MDR-TB in children compared to adults varied widely between countries. In Germany, Namibia, South Africa, the UK and the USA, MDR-TB was positively associated with age $<15$ years. In the remaining countries no association was established.

Despite the limitations intrinsic to the use of surveillance data and to the challenges of diagnosing childhood tuberculosis, our analysis suggests that proportions of MDR-TB in children and adults are similar in many settings. Of particular concern is the association found between age $<15$ years and MDR-TB in southern African countries with high HIV prevalence.

@ERSpublications

Surveillance data from 35 countries suggest that proportions of MDR-TB in children are not lower than those in adults http://ow.ly/kPgPH

Received: Nov 012012 | Accepted after revision: Dec 242012 | First published online: Dec 062012

Support statement: This study was supported by the United States Agency for International Development through a grant to the World Health Organization (US-2013-0579). The funders had no role in study design, data collection and analysis, decision to publish, or preparation of the manuscript.

Conflict of interest: None declared.

ERJ Open articles are open access and distributed under the terms of the Creative Commons Attribution NonCommercial Licence 3.0.

This article was modified in April 2016 to correct errors in the licence information. 


\section{Introduction}

Drug-resistant tuberculosis and multidrug-resistant tuberculosis (MDR-TB) in particular represent a major threat to the fight against tuberculosis globally. MDR-TB is defined as disease caused by strains of Mycobacterium tuberculosis resistant to at least isoniazid and rifampicin, the two most powerful first-line antituberculosis drugs. Among the world's 12 million (range 11-13 million) prevalent cases of tuberculosis estimated by the World Health Organization (WHO) in 2011, the best estimate was that 630000 (5.3\%) had MDR-TB [1]. Globally, most cases of MDR-TB remain undetected and untreated because of limited laboratory capacity to conduct tests for drug resistance and limited access to second-line treatment, which is very long (20 months is recommended for most cases according to current WHO guidelines) as well as toxic and expensive [1-3].

As with other forms of tuberculosis, MDR-TB can affect people of all age groups, including children (aged 0-14 years). However, very little is known about the magnitude of this problem in children [4]. The diagnosis of MDR-TB is bacteriological by definition, based on the isolation of strains resistant to medicines. While isolating M. tuberculosis in adults with pulmonary tuberculosis is generally an easy procedure (the exception is patients who are immunocompromised), children mainly have paucibacillary disease, which means that specimens for culture and drug susceptibility testing are often difficult to obtain, particularly from the youngest who cannot expectorate sputum [5]. In most cases adults represent the source of infection for children but household contact investigations are rarely performed as part of routine surveillance activities in resource-limited settings with a high burden of tuberculosis and tend to be confined to special research projects $[6,7]$. Therefore, the results of drug susceptibility testing from adult source cases are not systematically used to investigate drug resistance in children [5].

For these reasons published reports of drug-resistant tuberculosis in children are largely from hospital settings [8-11] where more efforts can be made to obtain respiratory specimens by gastric aspirates, induced sputum and/or nasopharyngeal aspirates, and bronchoalveolar lavage.

The objectives of the analyses presented in this manuscript are to describe all the available data on drugresistant tuberculosis among children collected by the WHO Global Project on Anti-tuberculosis Drug Resistance Surveillance between 1994 and 2011, and to assess the evidence whether children (aged $<15$ years) are more or less likely than adults (aged $\geqslant 15$ years) to have MDR-TB in each country for which data are available.

\section{Methods}

Since 1994 aggregated drug resistance surveillance data have been reported on an annual basis to WHO, which ascertains the quality and representativeness of the data, performs analyses and disseminates the findings on regular basis $[1,12,13]$. Three main principles are used to ensure data quality: 1) data should be representative of the patients with tuberculosis in the country/geographical setting under study; 2) patients' treatment histories should be carefully obtained and available medical records reviewed to clearly determine whether patients have or have not previously received antituberculosis drugs; and 3) laboratory methods for antituberculosis drug susceptibility testing should be selected from among those recommended by WHO, and all laboratory processes should be quality-assured in cooperation with a partner supranational reference laboratory $[14,15]$. Drug resistance surveillance data can be collected via special surveys of a representative sample of patients or continuous surveillance based on routine diagnostic drug susceptibility testing. In both cases, all consecutive patients with bacteriologically confirmed (smear and/or culture positive) pulmonary tuberculosis are included. Those with extrapulmonary disease and those who are already on treatment for tuberculosis are excluded. In special surveys, in order to select a sample of patients representative of all patients in a geographic setting, either of the following sampling techniques is used: $100 \%$ sampling of all diagnostic centres or probability-proportional-to-size cluster sampling [14]. In routine surveillance, in order to ensure representativeness of the data, the coverage of culture and drug susceptibility testing should be high. A detailed description of the criteria used by WHO to ascertain whether survey and continuous surveillance data meet quality and representativeness standards is provided elsewhere $[1,16]$.

Surveillance data used in this analysis were reported to WHO disaggregated by history of treatment (new or previously treated), sex (male or female) and age $(<15$ or $\geqslant 15$ years).

The full database of the Global Project on Anti-tuberculosis Drug Resistance Surveillance was investigated for this study. Subnational level data that were not representative of the entire country were excluded from the analysis. Data on susceptibility to isoniazid and rifampicin were reviewed and proportions of MDR-TB in children aged $<15$ years and adults aged $\geqslant 15$ years were compared. To assess the relationship between MDR-TB and age group, odds ratios with $95 \%$ confidence intervals were calculated using logistic regression models run separately for each country. For countries with data from multiple years, robust standard errors 
were calculated to account for within-country time dependencies and the longitudinal facet of the data. Stata (version 12; StataCorp, College Sta, TX, USA) was used for all analyses.

\section{Results}

Since 1994 data on susceptibility to isoniazid and rifampicin disaggregated by age group have been reported to WHO from continuous surveillance or special surveys from a total of 85 countries. Among them, 35 countries reported at least one case of MDR-TB in children $<15$ years old during the period 2000-2011. No country reported MDR-TB in children in the period 1994-1999. Figure 1 shows the 85 countries that reported drug resistance surveillance data by age group as well as the 35 countries that had at least one case of MDR-TB in children. A total of 323046 patients with tuberculosis who received drug susceptibility testing for isoniazid and rifampicin in these 35 countries, including 6070 children aged $<15$ years $(2 \%$ of all those tested), were included in the analysis. For most countries $(28(80 \%)$ out of 35$)$, data were generated from continuous surveillance systems and for two-thirds (23 (66\%) out of 35), data were available for more than 1 year. Coverage of drug susceptibility testing in culture-positive cases was $86-100 \%$ in all age groups except for Bulgaria, Georgia, Hungary and South Africa (range 36-72\%). Table 1 summarises the characteristics of the data reported.

Odds ratios $(95 \% \mathrm{CI})$ for MDR-TB in children aged $<15$ years compared to adults aged $\geqslant 15$ years varied widely among countries, from $0.25(0.02-3.34)$ to 5.76 (0.76-43.98). In five countries children aged $<15$ years with tuberculosis had significantly higher odds of harbouring MDR-TB strains compared to adult patients aged $\geqslant 15$ years (Germany: 1.58 (1.03-2.44); Namibia: 3.44 (1.13-10.51); South Africa: 1.52 (1.33-1.74); UK: 2.36 (1.60-3.49); USA: 2.35 (1.43-3.85). In Australia, a weak positive association between MDR-TB and age $<15$ years was documented (1.86 (0.98-3.53)). In the remaining 29 countries, no association between MDR-TB and age could be established (fig. 2).

Among the 36 countries included in our analysis, 10 (Austria, Belgium, Germany, Latvia, Namibia, Norway, Republic of Moldova, Sweden, UK and USA) reported at least one case of MDR-TB in children aged $<5$ years and in children aged 5-14 years. In these countries the odds of harbouring MDR-TB was not significantly different between these two groups of children.

\section{Discussion}

This study is the first analysis of levels of MDR-TB among children using a very large dataset that has been developed over 10 years by the WHO Global Project on Anti-tuberculosis Drug Resistance Surveillance. The results suggest that, in a given setting, the proportion of MDR-TB in children and adults with bacteriologically confirmed tuberculosis is broadly similar, a finding consistent with reports from a few small-scale surveys conducted in the Central African Republic, India, South Africa and the USA [11, 18-20]. In none of the countries in our study was the risk of MDR-TB in children significantly lower than in adults. At the same time, levels of MDR-TB in children are highly variable among countries, but insufficient data on potential risk factors mean that further research is needed to understand this heterogeneity.

The global dataset that we analysed illustrates that drug resistance in children is reported mainly by highincome countries with continuous surveillance systems based on routine diagnostic drug susceptibility

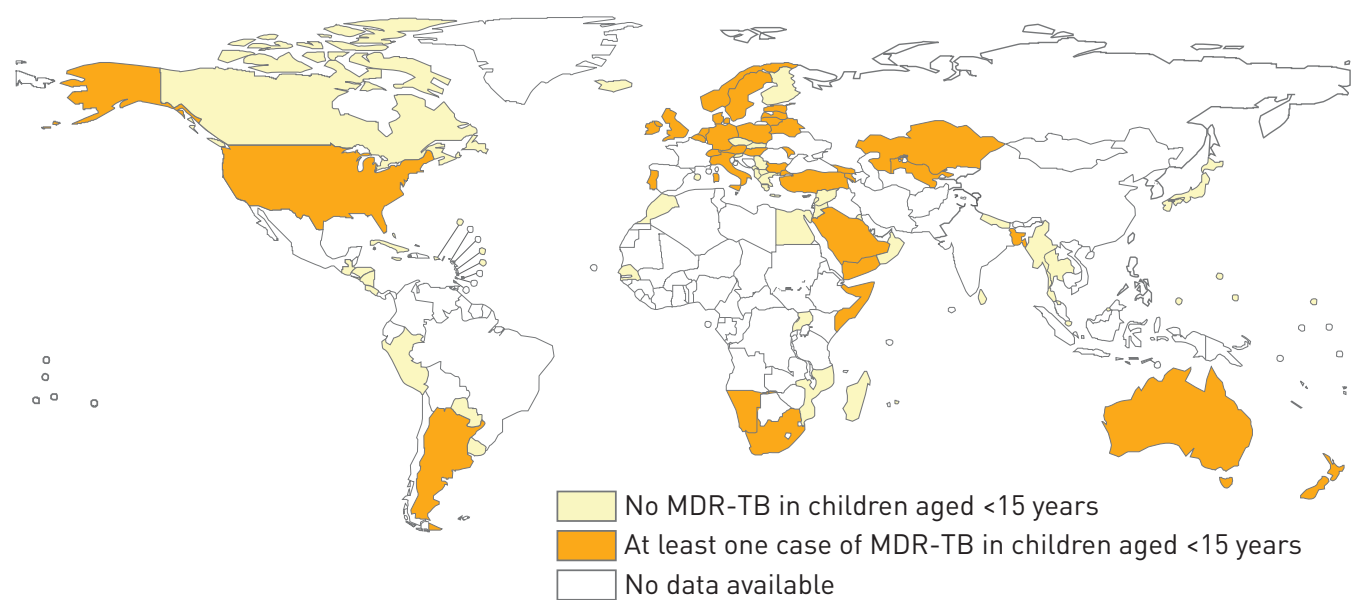

FIGURE 1 Countries that reported drug resistance survey/surveillance data disaggregated by age group, 2000-2011. MDR-TB: multidrug-resistant tuberculosis. 


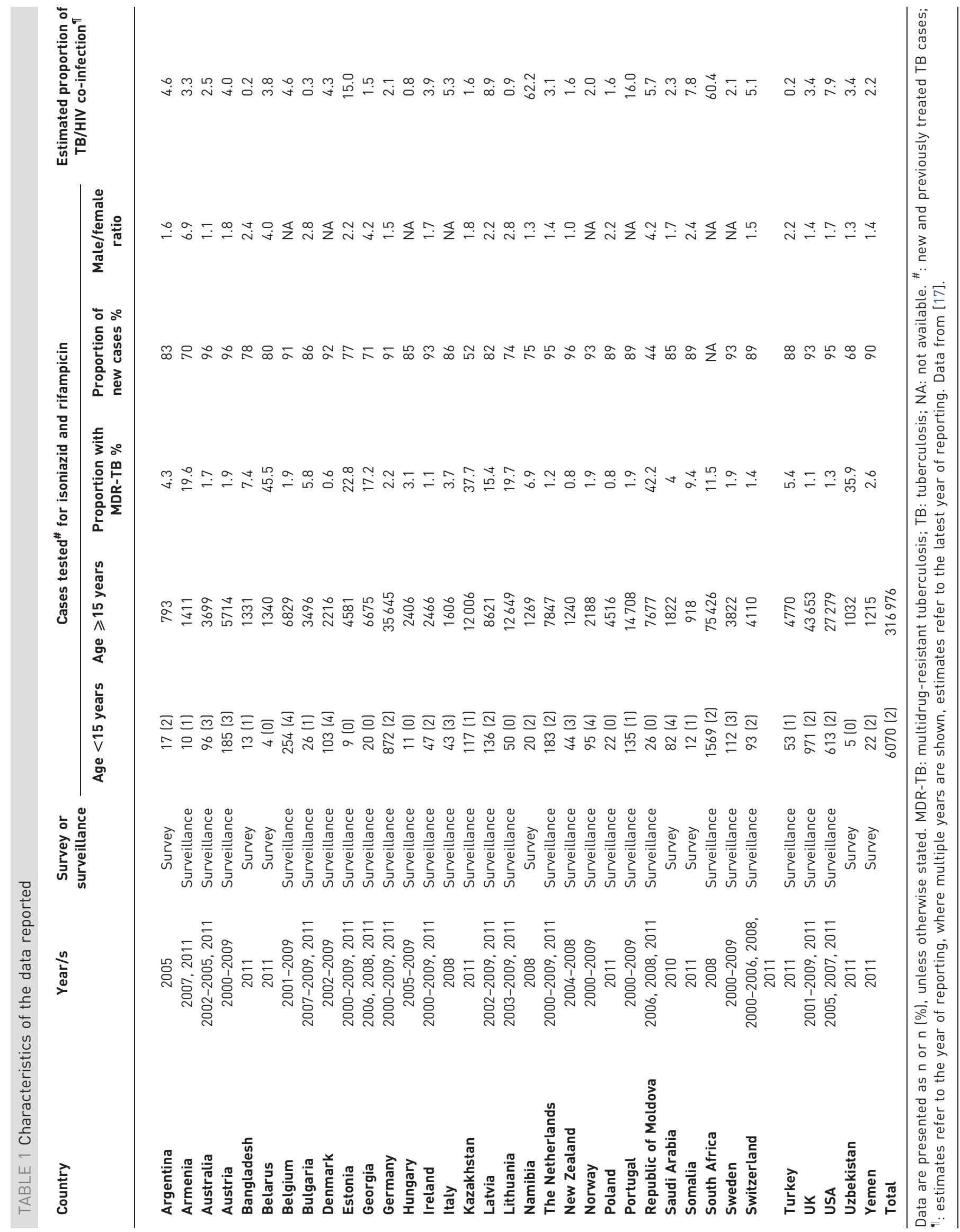




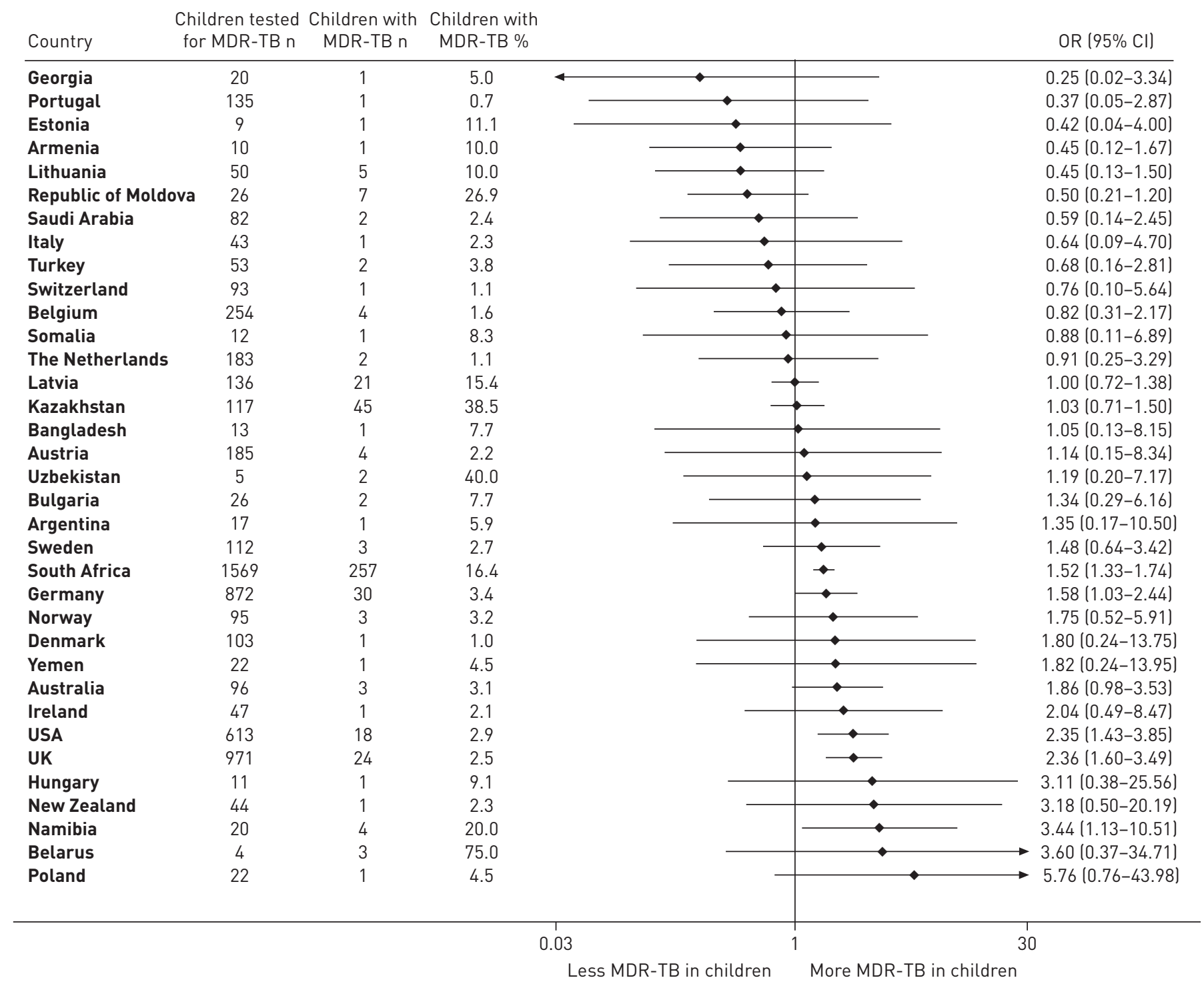

FIGURE 2 Forest plot depicting the association between multidrug-resistant tuberculosis (MDR-TB) in children (aged $<15$ years) versus adults (aged $\geqslant 15$ years) in countries reporting at least one MDR-TB case in children. Data among all (new and retreated) cases are presented.

testing of all bacteriologically confirmed tuberculosis cases. Continuous surveillance represents the most appropriate approach to monitoring of drug resistance [15], but it requires good laboratory infrastructure that is generally not available in resource-limited settings. Since the countries included in our analysis are a convenient sample and not representative of global regions, pooled estimates of the burden of MDR-TB in children have not been calculated.

Only seven countries were able to identify MDR-TB in children through special surveys conducted among representative samples of patients with tuberculosis. Surveys are generally not designed to accurately measure MDR-TB in a selected group of patients, such as children, as this would require very large sample sizes. In addition, obtaining ethical clearance for surveys involving children is very complex in some settings and this is sometimes used as a justification for excluding children from enrolment in surveys.

Novel survey approaches in screening, sampling and diagnosis that would allow the measurement of drug resistance in children in countries with limited laboratory capacity need to be explored. A new molecular technology has been recently introduced for the rapid diagnosis of tuberculosis and tuberculosis resistant to rifampicin [21]. The tool, named Xpert MTB/RIF assay (Cepheid, Sunnyvale, CA, USA), has the potential to facilitate the diagnosis of drug resistance in smear-negative patients, including in children. Two studies have suggested that the Xpert MTB/RIF assay can facilitate the diagnosis of drug resistance in children [22, 23], but more work should be done to improve the sensitivity of the test in patients with paucibacillary disease. 
Among the countries included in our analysis, a particular concern is the elevated risk of MDR-TB in children documented in Namibia and South Africa, two southern African countries with a high prevalence of HIV. If confirmed by further data, this finding has important implications for tuberculosis and MDR-TB control. As it is rare for children to develop drug resistance during their treatment [9], detection of MDR$\mathrm{TB}$ in this age group is usually a sensitive indicator of recent transmission of drug-resistant strains from contacts present in the environment in which they live.

The fact that in Germany, the UK and USA children appear to have a significantly higher risk of MDR-TB compared to adults also requires further evaluation. Contact investigations that are routinely performed in these countries could have played a role in this finding. In these countries the epidemic of tuberculosis is largely driven by migration [24-27] and foreign-born patients with tuberculosis are known to have higher levels of drug resistance compared to those born in the country. In Western European countries more than half of children with tuberculosis are $<5$ years old [27]. This is a strong indication of household transmission and highlights the need for better prevention and diagnosis of tuberculosis and MDR-TB in children and in particular among the youngest. Research on effective chemoprophylaxis regimens for children exposed to patients with MDR-TB is important.

The fact that in our analysis children aged $<15$ years represented $2 \%$ of all cases who received drug susceptibility testing is in line with the proportion of children among all cases notified with tuberculosis in 2011 (2\%) [1]. Our analysis has two main limitations that are intrinsic to the use of programmatic surveillance data. First, in a few countries, data on the age of tuberculosis patients were missing ( $8 \%$ of patients who were tested for drug susceptibility in South Africa and 2-3\% in Namibia and Italy). Overall, missing data on age did not affect the interpretation of the results. Second, the inherent challenges involved in diagnosing tuberculosis and MDR-TB in children, described above, are likely to affect the validity of surveillance data. Although survey and surveillance data used for this analysis met the representativeness and quality criteria defined by WHO, it is not possible to completely rule out selection bias introduced by the difficulties in diagnosing tuberculosis in children and by contact investigations in countries where those are routinely performed. These limitations should be taken into consideration when interpreting the results of our study.

Our analysis from a subset of 10 countries showed no significant difference in levels of resistance between children aged $<5$ years and children aged 5-14 years. This reinforces the hypothesis that, although obtaining specimens for culture from the youngest children is often difficult and drug-resistant tuberculosis is more likely to be underdiagnosed, younger children have a similar likelihood of harbouring drug resistant strains than older children. Given the limited availability of data for this analysis this finding should be confirmed by larger studies.

In conclusion, this analysis suggests that while the problem of MDR-TB in children is largely unknown and often unreported $[4,5,11,28]$, a child with tuberculosis is as likely as an adult with tuberculosis to have MDR-TB. To improve the identification of MDR-TB in children for treatment and for surveillance purposes, household contact investigation of all patients with MDR-TB should be systematically implemented and should always include children [29, 30]. In addition, children should be systematically included in all surveillance activities, including drug resistance surveys when routine surveillance is not in place. More broadly, the data illustrate the need for intensified efforts to develop better tests for the diagnosis of tuberculosis and MDR-TB in children and to improve the availability of paediatric drug formulations for the treatment of children with tuberculosis and MDR-TB.

\section{Acknowledgements}

All authors are staff members of the World Health Organization. The authors alone are responsible for the views expressed in this publication and they do not necessarily represent the decisions or policies of the World Health Organization.

\section{References}

World Health Organization. Global Tuberculosis Report 2012. Geneva, WHO Press, 2012.

Falzon D, Jaramillo E, Schünemann HJ, et al. WHO guidelines for the programmatic management of drug-resistant tuberculosis: 2011 update. Eur Respir J 2011; 38: 516-528.

Falzon D, Gandhi N, Migliori GB, et al. Resistance to fluoroquinolones and second-line injectable drugs: impact on MDR-TB outcomes. Eur Respir J 2013; 42: 156-168.

4 Marais BJ, Schaaf HS. Childhood tuberculosis: an emerging and previously neglected problem. Infect Dis Clin North Am 2010; 24: 727-749.

5 Seddon JA, Hesseling AC, Willemse M, et al. Culture-confirmed multidrug-resistant tuberculosis in children: clinical features, treatment, and outcome. Clin Infect Dis 2012; 54: 157-166.

6 Hwang TJ, Ottmani S, Uplekar M. A rapid assessment of prevailing policies on tuberculosis contact investigation. Int J Tuberc Lung Dis 2011; 15: 1620-1623. 
7 Hill PC, Ota MO. Tuberculosis case-contact research in endemic tropical settings: design, conduct, and relevance to other infectious diseases. Lancet Infect Dis 2010; 10: 723-732.

8 Schaaf HS, Marais BJ, Hesseling AC, et al. Childhood drug-resistant tuberculosis in the Western Cape Province of South Africa. Acta Paediatr 2006; 95: 523-528.

9 Schaaf HS, Marais BJ, Hesseling AC, et al. Surveillance of antituberculosis drug resistance among children from the Western Cape Province of South Africa - an upward trend. Am J Public Health 2009; 99: 1486-1490.

10 Kassa-Kelembho E, Bobossi-Serengbe G, Takeng EC, et al. Surveillance of drug-resistant childhood tuberculosis in Bangui, Central African Republic. Int J Tuberc Lung Dis 2004; 8: 574-578.

11 Fairlie L, Beylis NC, Reubenson G, et al. High prevalence of childhood multi-drug resistant tuberculosis in Johannesburg, South Africa: a cross sectional study. BMC Infect Dis 2011; 11: 28.

12 Zignol M, van Gemert W, Falzon D, et al. Surveillance of anti-tuberculosis drug resistance in the world: an updated analysis, 2007-2010. Bull World Health Organ 2012; 90: 111D-119D.

13 Wright A, Zignol M, Van Deun A, et al. Epidemiology of antituberculosis drug resistance 2002-07: an updated analysis of the Global Project on Anti-Tuberculosis Drug Resistance Surveillance. Lancet 2009; 373: 1861-1873.

14 World Health Organization. Guidelines for Surveillance of Drug Resistance in Tuberculosis. 4th Edn. Publication number: WHO/HTM/TB/2009.422. Geneva, WHO Press, 2009.

15 Zignol M, van Gemert W, Falzon D, et al. Modernizing surveillance of antituberculosis drug resistance: from special surveys to routine testing. Clin Infect Dis 2011; 52: 901-906.

16 World Health Organization. Multidrug and Extensively Drug-resistant TB (M/XDR-TB): 2010 Global Report on Surveillance and Response. Publication number: WHO/HTM/TB/2009.422 Geneva, WHO, 2010.

17 World Health Organization. TB Data. www.who.int/tb/country/data/download/en/index.html Date last accessed: November 1, 2012. Date last updated: November 1, 2012.

18 Swaminathan S, Datta M, Radhamani MP, et al. A profile of bacteriologically confirmed pulmonary tuberculosis in children. Indian Pediatr 2008; 45: 743-747.

19 Schaaf HS, Vermeulen HA, Gie RP, et al. Evaluation of young children in household contact with adult multidrugresistant pulmonary tuberculosis cases. Pediatr Infect Dis J 1999; 18: 494-500.

20 Steiner M, Cosio A. Primary tuberculosis in children. 1. Incidence of primary drug-resistant disease in 332 children observed between the years 1961 and 1964 at the Kings County Medical Center of Brooklyn. N Engl J Med 1966; 274: 755-759.

21 Boehme CC, Nabeta P, Hillemann D, et al. Rapid molecular detection of tuberculosis and rifampin resistance. N Engl J Med 2010; 363: 1005-1015.

22 Nicol MP, Workman L, Isaacs W, et al. Accuracy of the Xpert MTB/RIF test for the diagnosis of pulmonary tuberculosis in children admitted to hospital in Cape Town, South Africa: a descriptive study. Lancet Infect Dis 2011; 11: 819-824.

23 Rachow A, Clowes P, Saathoff E, et al. Increased and expedited case detection by Xpert MTB/RIF assay in childhood tuberculosis: a prospective cohort study. Clin Infect Dis 2012; 54: 1388-1396.

24 Tuberculosis in the UK. London, Health Protection Agency, 2011. www.hpa.org.uk/webc/HPAwebFile/HPAweb_C/ 1317131791612 Date last accessed: November 1, 2012. Date last updated: December 2011.

25 Dara M, de Colombani P, Petrova-Benedict R, et al. The minimum package for cross-border TB control and care in the WHO European region: a Wolfheze consensus statement. Eur Respir J 2012; 40: 1081-1090.

26 Moore M, Onorato IM, McCray E, et al. Trends in drug-resistant tuberculosis in the United States, 1993-1996. JAMA 1997; 278: 833-837.

27 European Centre for Disease Prevention and Control/World Health Organization Regional Office for Europe. Tuberculosis Surveillance and Monitoring in Europe 2012. Stockholm, European Centre for Disease Prevention and Control, 2012.

28 Ettehad D, Schaaf HS, Seddon JA, et al. Treatment outcomes for children with multidrug-resistant tuberculosis: a systematic review and meta-analysis. Lancet Infect Dis 2012; 12: 449-456.

29 Becerra MC, Appleton SC, Franke MF, et al. Tuberculosis burden in households of patients with multidrugresistant and extensively drug-resistant tuberculosis: a retrospective cohort study. Lancet 2011; 377: 147-152.

30 Schaaf HS, Marais BJ. Management of multidrug-resistant tuberculosis in children: a survival guide for paediatricians. Paediatr Respir Rev 2011; 12: 31-38. 